\title{
Loss of DSTYK activates Wnt/ $\beta$-catenin signaling and glycolysis in lung adenocarcinoma
}

\author{
Chenxi Zhong ${ }^{1,3}$, Ming Chen ${ }^{1,3}$, Yu Chen ${ }^{2,3}$, Feng Yao (D) ${ }^{1 凶}$ and Wentao Fang ${ }^{1 凶}$ \\ (c) The Author(s) 2021
}

\begin{abstract}
Aberrant activation of $\mathrm{Wnt} / \mathrm{\beta}$-catenin signaling and dysregulation of metabolism have been frequently observed in lung cancer. However, the molecular mechanism by which Wnt/ $\beta$-catenin signaling is regulated and the link between Wnt/ $\beta$-catenin signaling and cancer metabolism are not fully understood. In this study, we showed that the loss of dual serine/threonine tyrosine protein kinase (DSTYK) led to the activation of Wnt/ $\beta$-catenin signaling and upregulation of its target gene, lactate dehydrogenase (LDHA), and thus the elevation of lactate. DSTYK phosphorylated the $\mathrm{N}$-terminal domain of $\beta$-catenin and inhibited Wnt/ $\beta$-catenin signaling, which led to the inhibition of cell growth, colony formation and tumorigenesis in a lung adenocarcinoma mouse model. DSTYK was downregulated in lung cancer tissues, and its expression was positively correlated with the survival of patients with lung adenocarcinoma. Taken together, these results demonstrate that the loss of DSTYK activates Wnt/ $\beta$-catenin/LDHA signaling to promote the tumorigenesis of lung cancer and that DSTYK may be a therapeutic target.
\end{abstract}

Cell Death and Disease (2021)12:1122; https://doi.org/10.1038/s41419-021-04385-1

\section{INTRODUCTION}

Lung cancer is one of the most common malignant cancers [1, 2] and exploring its pathogenesis is of great significance for early diagnosis and treatment [3]. During the oncogenesis of lung cancer, many signals involved in embryonic development are activated, such as the Wnt/ $\beta$-catenin, TGF $\beta$, and Hedgehog pathways [4-6]; an abnormal metabolism is one of the basic characteristics of tumor cells [7]. Therefore, investigating the regulatory mechanisms of these pathways and their effects on metabolism is vital for the design of intervention strategies.

The Wnt/ $\beta$-catenin pathway plays an important role in the progression of lung cancer [8,9]. A constitutively activating mutation of $\beta$-catenin was found in clinical specimens of lung cancer, which activated a signaling pathway in which $\beta$-catenin is the core element and resulted in tumorigenesis [10]. When cells are in a resting state, a degradation complex composed of Axin, $A P C, G S K 3 \beta$, and CK1a within the cytoplasm phosphorylates $\beta$-catenin [11]. Phosphorylated $\beta$-catenin is bound by the E3 ligase $\beta-\operatorname{TrCP}$, ubiquitinated and degraded through the proteasomal pathway. Phosphorylation of $\beta$-catenin at serine/threonine residues $33,37,41$, and 45 of its $\mathrm{N}$-terminal domain is the core event in $\beta$-catenin regulation [12]. Currently, GSK3 $\beta$ and CK1 $a$ are thought to be responsible for the phosphorylation of these sites during individual embryonic development. CK1a phosphorylates serine residue 45 before GSK3 $\beta$ phosphorylates residues 33, 37, and 41 [13]. The novel kinases that phosphorylate these amino acids in the context of tumor progression remain undetermined.

Dual serine/threonine and tyrosine protein kinase (DSTYK) encodes a protein product consisting of 850-931 amino acids
[14]. The N-terminal domain of DSTYK is a noncatalytic domain with low conservation among species [14]. In contrast, its C-terminus is the kinase catalytic domain and shows high conservation among species [14]. DSTYK is expressed in the brain, heart, muscles, kidneys and lungs and is distributed in the cytoplasm and nucleus [14].

The deletion of DSTYK in zebrafish via positional cloning was found by CRISPR-Cas 9 screening to cause notochord development defects $[15,16]$. Moreover, in a study of its molecular mechanisms, DSTYK was found to be necessary for lysosome synthesis in mammalian cells, promoting fusion of the late endosome and lysosomal system $[15,16]$. Furthermore, DSTYK inhibited $m$ TORC1, accelerated TFEB entry into the nucleus, and activated the expression of genes related to lysosome synthesis $[15,16]$.

Whole-exon sequencing showed that DSTYK exhibits multiple mutations in a variety of pathological conditions. DSTYK gene mutations have also been observed in unilateral renal agenesis [17]. In addition, DSTYK mutation is closely related to urinary tract deformity $[18,19]$. A water maze experiment demonstrated that deletion of the DSTYK kinase domain seriously affected the learning capabilities and memory functions of mice [20]. Furthermore, a mutated DSTYK (Met296/le) in intracranial solitary fibrous tumors promoted tumor metastasis by activating the ERK pathway [21]. To date, the function and mechanism of DSTYK in tumorigenesis remain unclear.

In this study, the expression pattern of DSTYK in lung adenocarcinoma was detected and the function and expression pattern of DSTYK in lung cancer were evaluated. Moreover, the biological function mechanism of DSTYK was investigated.

\footnotetext{
${ }^{1}$ Department of Thoracic Surgery, Shanghai Chest Hospital, Shanghai Jiao Tong University, 200030 Shanghai, China. ${ }^{2}$ Department of Thoracic Surgery, Shandong Zaozhuang

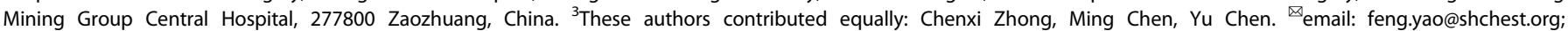
vwtfang@hotmail.com

Edited by Professor Stephen Tait
}

Received: 1 November 2020 Revised: 3 August 2021 Accepted: 16 September 2021

Published online: 01 December 2021 


\section{MATERIALS AND METHODS}

\section{Cell culture and transfection}

Normal bronchial epithelial cell line Bease-2B and lung cancer cell lines were obtained from the Cell Bank of the Chinese Academy of Science (Shanghai, China) and maintained in DMEM (Gibco) containing 10\% FBS (Gibco) and antibiotics in a $5 \% \mathrm{CO}_{2}$ incubator. Cells were transfected with Lipofectamine 8000 (Invitrogen) according to manufacturer instructions. Stable cell lines were selected using puromycin (Sangon Biotechnology, A610593) for one week. Cell lines were authenticated using STR profile analysis and used within 3-20 passages of thawing the original stocks.

\section{qPCR}

Total RNA was extracted from cells or tissues using TRlzol and reverse transcribed to CDNA using a kit (Takara). RT-PCR analysis was performed using an Applied Biosystems Prism 7900 HT sequence detection system with Universal SYBR qPCR Master Mix (Vazyme, China). The expression levels of each mRNA relative to the $\beta$-actin expression level were calculated based on the $2^{-\Delta \Delta C t}$ method. The primer sequences were as follows: F, 5'-ttgcatactgatcctcgg-3'; R, 5'-tgtgcactagttcatact-3'.

\section{Immunohistochemistry (IHC)}

30 clinical samples were collected from Shanghai Chest Hospital after the informed consent was obtained was obtained from all subject. This study was approved by the ethical committee of the Shanghai Chest Hospital. The clinical information was listed in Table S1. Tissues were embedded in paraffin and cut into $5 \mu \mathrm{m}$ sections. Dewaxing and rehydration were performed by placing slides in a decreasing gradient series of xylol and ethanol. Antigen recovery was performed by incubating the sections in a $\mathrm{pH} 6.0$ citrate sodium solution at $100^{\circ} \mathrm{C}$ for $20 \mathrm{~min}$. After blocking endogenous peroxidase activity using a kit (ZSGB-BIO, PV-8000), the tissues were incubated with primary antibody (anti-DSTYK, 1;100, Proteintech, 20102-1-AP; anti- $\beta$-catenin, $1 ; 100$, CST, 8480 ; or anti-LDHA, 1;100, Proteintech, 19987-1-AP) at $4{ }^{\circ} \mathrm{C}$ overnight. Then, secondary antibody was added, and the tissues were incubated at room temperature for $1 \mathrm{~h}$. The signals were developed using DAB (ZSGB-Bio, Beijing, China), and the sections were stained with hematoxylin. Tissues were examined under a microscope, and scoring was performed as described.

\section{KP mouse model}

p53 ${ }^{f / f}$; LSL-Kras ${ }^{G 12 D}$ (KP) model C57BL/6 mice were housed under standard conditions with $12 \mathrm{~h}$ dark and $12 \mathrm{~h}$ light cycles.

To study the roles of DSTYK in the tumorigenesis in vivo, the sg con or sg DSTYK were inserted into the cassette of PSECC lentivirus vector which expressed cas 9 enzyme and Cre recombinase. To induce lung cancer, the 8-week-old male KP mice were randomly divided into two groups (5 mice in each group) and anesthetized with $2.5 \%$ avertin; full anesthetization and absence of reaction to pain were ensured. A total of $1 \times 10^{9}$ viruses (OBIO, Shanghai) were administered per mouse ( 8 weeks old) using an intranasal/ orthotropic infection protocol as described [22]. The administration of $\mathrm{pSECC} / \mathrm{Sg}$ con viruses led to the deletion of P53 and activation of Kras ${ }^{\mathrm{G} 12 \mathrm{D}}$. The administration of pSECC/Sg DSTYK viruses led to the deletion of P53 and DSTYK and activation of Kras ${ }^{G 12 D}$. Twelve weeks later, the lungs were harvested, and western blot assays were performed.

For the examination of DSTYK protein levels in the KP tumors, the mice were treated with/without Ad-Cre virus (adenovirus). This study was approved by the ethical committee of the Shanghai Chest Hospital, and complied with the ethical regulations of ethical committee of the Shanghai Chest Hospital.

\section{Western blot}

Cells were harvested with RIPA buffer. The protein concentration was determined using BCA. Then, SDS-PAGE was performed, and the proteins were transferred to a PVDF membrane. After blocking with 5\% BSA at room temperature for $1 \mathrm{~h}$, the membrane was incubated with the primary antibody for $4 \mathrm{~h}$ and sequentially with the secondary antibody for $1 \mathrm{~h}$. Then, the signals were examined using an ECL kit. The information about the antibodies were: Anti-DSTYK (Proteintech, 20102-1-AP), anti-GAPDH (Santa Cruz, sc-47724), ant- $\beta$-catenin (CST, 8480), anti-phosphorylated $\beta$-catenin (CST, 9561), anti-GST (Santa Cruz, sc-138), anti-Flag (Proteintech, 80010-1-RR), anti-Cyclin D1 (Santa Cruz, sc-8396), anti-c-Jun (Santa Cruz, sc74543), anti-c-Myc (Santa Cruz, sc-40), anti-LDHA (Proteintech, 19987-1-AP), anti-Tubulin (Santa Cruz, sc-166729).

\section{MTT assay}

An MTT (Merck, 11465007001) kit was used. Cells were plated in 96-well plates at a density of 1000 cells per well, and each well was loaded with $200 \mu \mathrm{l}$ of medium. On days 1, 3, 5, and 7, $20 \mu \mathrm{l}$ of MTT solution were added to each well, and the plates were incubated for $4 \mathrm{~h}$. Then, supernatants were removed, precipitates were dissolved in $200 \mu \mathrm{l}$ of DMSO, and absorbance was measured.

\section{Colony formation assay}

Cells were suspended in $2 \times$ DMEM at a density of $10^{6}$ cells $/ \mathrm{L}$. A bottom agar layer was prepared with an equal volume of $1.2 \%$ agar and $2 \times$ DMEM containing antibiotics and $20 \%$ FBS, and $3 \mathrm{ml}$ of the mixture was added to a $6 \mathrm{~cm}$ dish. Thirty minutes later, a bottom agar layer was prepared with an equal volume of $1.2 \%$ agar and $2 \times$ DMEM with an additional $0.2 \mathrm{ml}$ of the cell suspension. After softly mixing, the mixture was added to the dish with the bottom agar layer. Fourteen days later, colonies were counted.

\section{Sphere formation assay}

Cells from each group were collected and digested into single cells. Subsequently, for each group, $2 \times 10^{4}$ viable cells per well were counted and seeded in low-adherent 6-well plates (Corning, USA) in serum-free F12/MEM containing B27, EGF $(20 \mathrm{ng} / \mathrm{ml})$ and FGF $(40 \mathrm{ng} / \mathrm{ml})$. After incubation at $37^{\circ} \mathrm{C}$ for 14 days, pictures were taken under a microscope, and tumor spheres in five separate fields were counted. EGF (R\&D, 236-EG), FGF (R\&D, 233-FB), and B27 (R\&D, AR008) were obtained from R\&D.

\section{Immunoprecipitation}

Cells were scraped and proteins were extracted from the cells with $1 \mathrm{ml}$ RIPA buffer. After centrifugation $\left(4^{\circ} \mathrm{C}, 12,000 \mathrm{rpm}\right)$ for $20 \mathrm{~min}$, supernatant was divided into two tubes and immunoprecipitated with the indicated antibody and IgG as control at $4{ }^{\circ} \mathrm{C}$. On the next day, protein A beads (Sangon, Shanghai) were added to the supernatant and incubated for another $4 \mathrm{~h}$. Then, the beads were collected through centrifugation and washed three times. After as much wash buffer as possible was removed, the beads were mixed with $30 \mu$ l of loading buffer and boiled for $5 \mathrm{~min}$ at $100^{\circ} \mathrm{C}$. The precipitates were examined using western blot.

\section{GST pull-down assay}

Cells were scraped, and proteins were extracted from the cells with $1 \mathrm{ml}$ RIPA buffer. After centrifugation $\left(4^{\circ} \mathrm{C}, 12,000 \mathrm{rpm}\right)$ for $20 \mathrm{~min}$, the supernatants were divided into two tubes and immunoprecipitated with $5 \mu \mathrm{g}$ of GST or GST-DSTYK fusion protein at $4{ }^{\circ} \mathrm{C}$. The next day, Sepharose $4 \mathrm{~B}$ beads (GE Healthcare) were added to the supernatants and incubated for another $4 \mathrm{~h}$. Then, the beads were collected through centrifugation and washed three times. After as much wash buffer as possible was removed, the beads were mixed with $30 \mu \mathrm{l}$ of loading buffer and boiled for $5 \mathrm{~min}$ at $100^{\circ} \mathrm{C}$. The precipitates were then examined using western blot.

\section{Reporter assay}

Cells at $70 \%$ confluence were plated in a 24-well plate. For each well, the cells were transfected with $0.01 \mu \mathrm{g}$ of TOPFlash, $0.01 \mu \mathrm{g}$ of TK Renilla, and $0.25 \mu \mathrm{g}$ of DSTYK expression vector or empty vector. Forty-eight hours later, the cells were lysed with RIPA buffer, and reporter activity was examined using a dual reporter assay kit (Promega).

\section{In vitro kinase assay}

Fusion protein GST- $\beta$-catenin (N) (the $N$-terminal domain of $\beta$-catenin) was purified and incubated with Flag-DSTYK protein immunoprecipitated from a stable A549 cell line in the presence of kinase assay buffer and ATP (CST, 9802). The reaction was performed at $32{ }^{\circ} \mathrm{C}$ for $30 \mathrm{~min}$ and stopped by the addition of loading buffer. The phosphorylation of $\beta$-catenin was examined using western blot.

\section{ChIP}

ChIP was performed using a kit (Cell Signaling Technology, 9004) according to manufacturer instructions. The primers for qPCR were as follows: F, 5'-TCAAAACCAAGAAACTCAG-3'; F， 5'-GGGAAAGACCAAATGATTA-3'. qPCR was performed using a $2 \times$ SYBR qPCR mixture according to manufacturer instructions. 


\section{Lactate measurement}

A lactate assay was performed using a kit (Abcam, ab65331). Briefly, cell culture medium was collected. The culture medium and standards were added to the wells of a plate, after which reaction mixture was added and incubated for $30 \mathrm{~min}$ at room temperature. The samples were analyzed with a microplate reader according to manufacturer instructions.

\section{RNA sequencing}

For RNA sequencing, A549 control cells and cells with the knockdown of DSTYK were harvested. The libraries were performed by Novogene (Tianjin, China), and an Illumina HiSeqX10 (Illumina, San Diego, CA, USA) was used for high-throughput sequencing. Quality control and removal of adapters of raw paired-end reads were performed using fastp (version 0.20.1). The gene expression counts were analyzed using HTseq-count software. The differently expressed genes were identified using DEseq2 (version 3.12) in R environment (version 3.6.3). Data were uploaded, and the GEO number is GSE178487.

\section{Statistical analysis}

All experiments in this study were performed in triplicate and error bars represent the standard deviation $( \pm$ S.D) of triplicate samples. Statistical analysis was conducted using GraphPad Prism (version 7.0). Comparisons between groups were performed using two-tailed independent sample
Student's $t$-tests analysis. Data were expressed as mean \pm S.D, $P<0.05$ was considered a significant difference $\left({ }^{*} P<0.05 ;{ }^{*} P<0.01 ;{ }^{* *} P<0.001\right)$.

\section{RESULTS}

Low DSTYK expression was observed in lung cancer

To clarify the expression pattern of DSTYK in lung cancer, we initially searched the Kmplot database for correlations between DSTYK expression and lung cancer patient survival. Bioinformatics analysis showed that DSTYK expression in lung cancer was positively correlated with patient survival time (Fig. 1A). Next, we detected the mRNA and protein levels of DSTYK in 30 lung cancer tissues and 30 paracancerous tissues by qPCR. The mRNA level of DSTYK was low in lung cancer (Fig. 1B), and immunohistochemistry indicated that the protein level of DSTYK was low in lung cancer (Fig. 1C, D). The KP (LSL-Kras ${ }^{G 12 D} ;$ P55 ${ }^{\text {loxp/loxp }}$ ) mouse model is a classic model for investigating lung cancer. The western blot (Fig. 1E, up) and $\mathrm{IHC}$ (Fig. 1E, below) results showed that DSTYK expression levels was decreased in the tumorigenesis of KP mice (the mice were treated with Ad-Cre virus to induce the tumor). Finally, DSTYK expression in lung cancer cell lines was detected by Western blot. As shown in Fig. 1F, DSTYK was highly expressed in
A

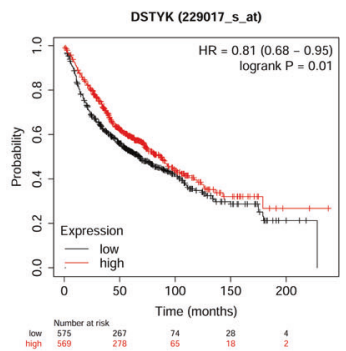

C

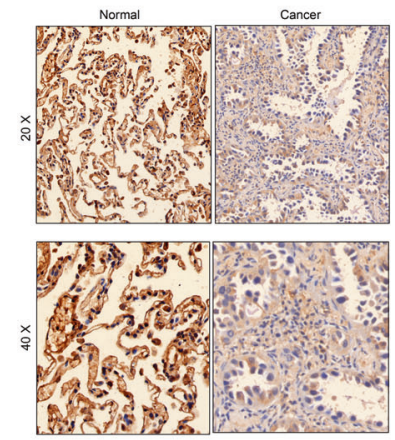

D

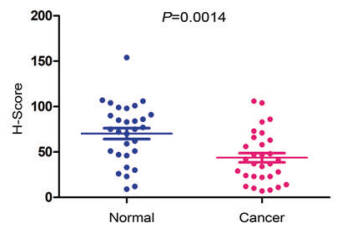

B

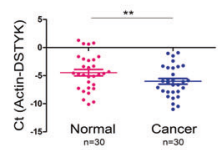

$\mathrm{E}$
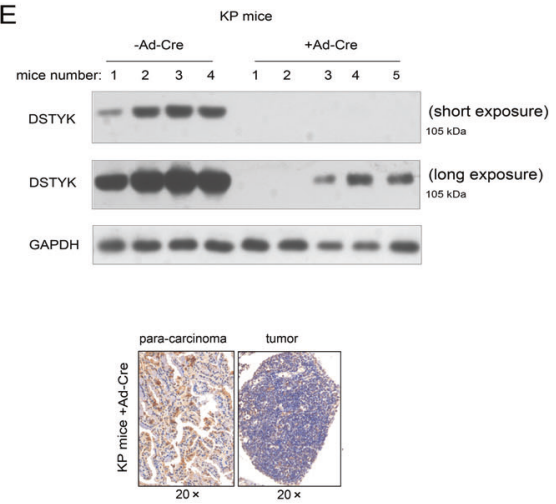

$\mathrm{F}$

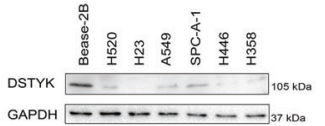

Fig. 1 DSTYK was downregulated in lung cancer. A The KmPlot database was mined to confirm the correlation between the expression of DSTYK and the survival of patients with lung cancer. B qPCR was performed to determine the mRNA levels of DSTYK in lung cancer tissues and noncancerous tissues. ${ }^{*} P<0.01$. C Immunohistochemistry $(\mathrm{IHC})$ was performed to determine the protein levels of DSTYK in lung cancer tissues and noncancerous tissues. The images with different magnification were shown and the scale bar was indicated. D Statistical analysis of the data in C. The IHC intensity was scored and analyzed. E Western blot and IHC were performed to determine the protein levels of DSTYK in the KP mouse model. Eight-week-old KP mice in the experimental group were treated with Ad-Cre virus ( $+\mathrm{Ad}-\mathrm{Cre})\left(10^{9}\right.$ per mouse) to delete the expression of $\mathrm{P} 53$ and activate the expression of Kras ${ }^{\mathrm{G} 12 \mathrm{D}}$. Mice in the control group were treated with control virus. Twelve weeks later, the mice were killed, lung tissues were collected, and DSTYK protein levels were examined by western blot (up) and IHC (below). The images of western blot with long exposure and short exposure were shown. $\mathbf{F}$ The protein levels of DSTYK in normal lung epithelial cells (Bease-2B) and lung cancer cells. 
the normal pulmonary bronchial epithelial cell line Bease- $2 B$ (Fig. 1F). These results indicate that DSTYK expression is downregulated in lung cancer.

\section{DSTYK inhibited the growth, colony formation, and sphere formation of lung cancer cells}

Downregulated DSTYK expression in lung cancer implies that DSTYK plays an important role in lung cancer progression. We overexpressed DSTYK in A549 and SPC-A-1 cells to further explore the function of DSTYK in the progression of lung cancer (Fig. 2A). Figure $2 \mathrm{~B}$ shows that DSTYK overexpression inhibited the growth of lung cancer cells in liquid medium. Anchorage-independent growth is a basic characteristic of tumor cells. Therefore, we evaluated the effect of DSTYK expression on the anchorageindependent growth of lung adenocarcinoma cells via soft agar colony formation and sphere formation assays. The results showed that DSTYK overexpression inhibited colony formation on soft agar (Fig. 2C, D) and sphere-forming abilities of lung cancer cells (Fig. 2E). However, when DSTYK expression was knocked down in A549 and SPC-A-1 cells (Fig. 3A), various functional tests indicated that DSTYK knockdown promoted growth (Fig. 3B) and colony formation (Fig. 3C, D) of A549 and SPC-A-1 cells. Moreover, knockout of the expression of DSTYK in the KP mouse model promoted tumorigenesis (Fig. $3 \mathrm{E}-\mathrm{H}$ ). These results confirm that DSTYK inhibits the progression of lung tumors.

Interaction between DSTYK and $\boldsymbol{\beta}$-catenin in lung cancer cells We detected the interactions among DSTYK and key components of the $\mathrm{Wnt} / \beta$-catenin and TGF $\beta$ signaling pathways to explore the molecular mechanism by which DSTYK regulates lung cancer and found an interaction between exogenous DSTYK expression and $\beta$-catenin (Fig. 4A). A GST pull-down test showed the same result (Fig. 4B). Moreover, endogenously expressed DSTYK in lung adenocarcinoma cells interacted with $\beta$-catenin (Fig. 4C), and an interaction between the $\mathrm{N}$-terminal domain of DSTYK and $\beta$-catenin was observed by immunoprecipitation (Fig. 4D).

\section{DSTYK phosphorylated $\beta$-catenin and inhibited the Wnt/ $\beta$-catenin signaling pathway}

We first investigated the effect of DSTYK expression on the TOPFlash reporter to elucidate the influence of DSTYK on the Wnt/ $\beta$-catenin signaling pathway. The results showed that DSTYK overexpression inhibited the Wnt3a-induced activation of the
A

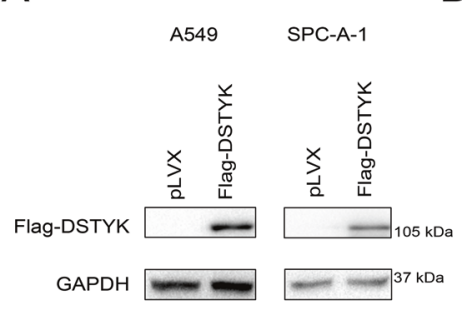

B

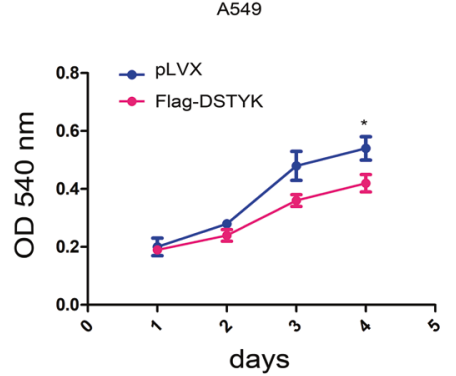

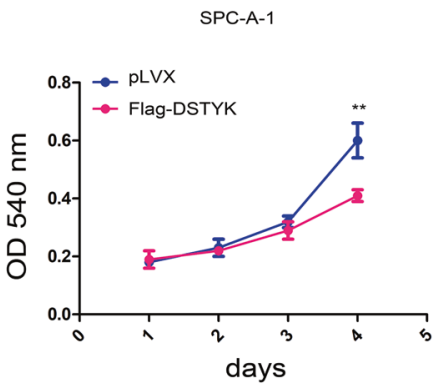

$E$

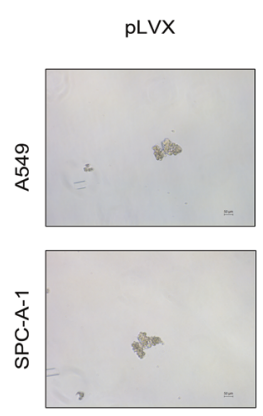

Flag-DSTYK
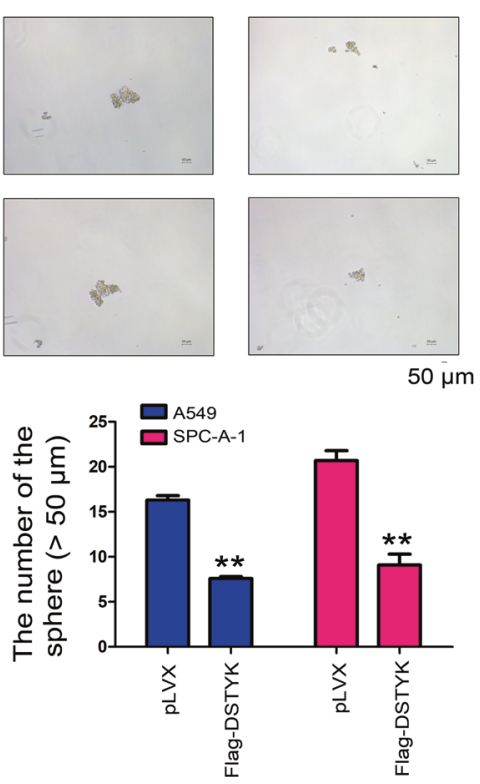

Fig. 2 DSTYK inhibited the growth of A549 and SPC-A-1 cells. A Western blot was performed to examine the overexpression of DSTYK. A549 and SPC-A-1 cells were infected with lentivirus expressing Flag-tagged DSTYK and selected with puromycin. Then, western blotting was performed. B The MTT assay was performed to examine the effects of DSTYK on the growth of A549 and SPC-A-1 cells. C, D A colony formation assay was performed, and the results were quantified. E Sphere formation was assessed to examine the roles of DSTYK in stemness. Details about sphere formation are described in the "Materials and methods". The spheres were photographed and counted. The scale bar was indicated. ${ }^{*} P<0.05 ;{ }^{* *} P<0.01$. 
A

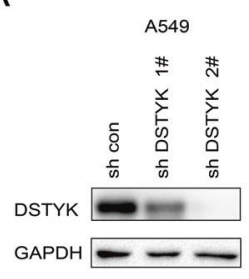

B

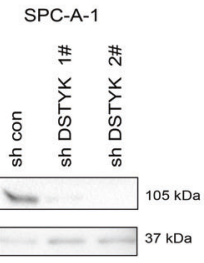

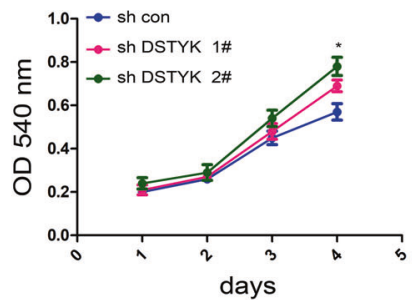

SPC-A-1

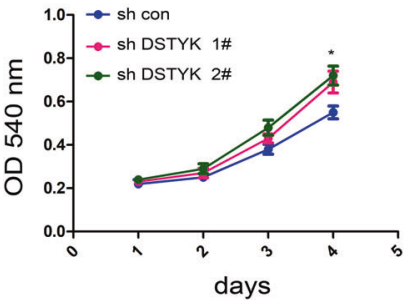

C

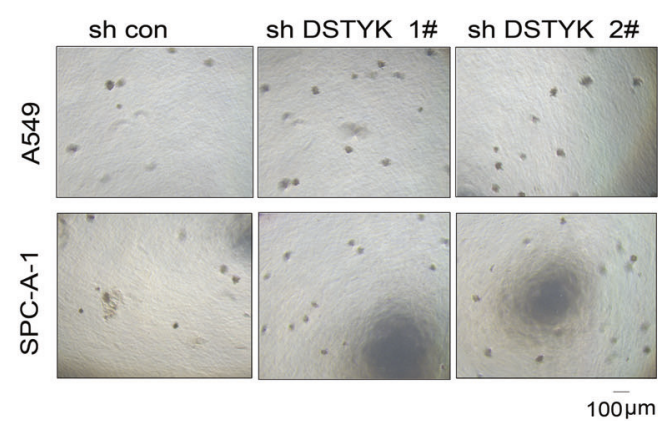

D

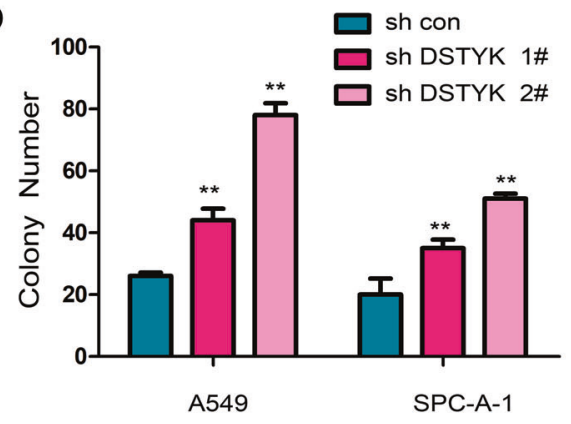

E Sg con

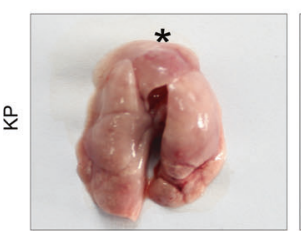

G

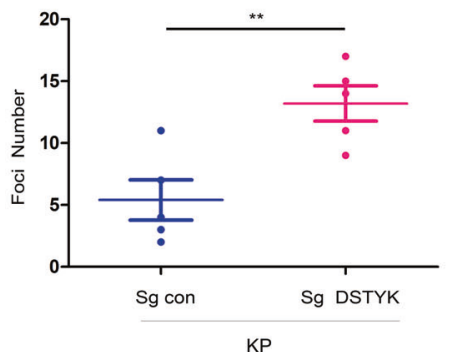

Sg DSTYK

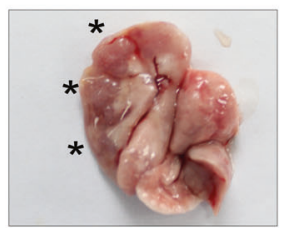

F

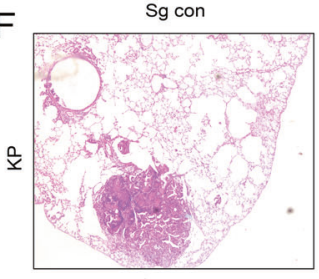

$4 \times$
Sg DSTYK

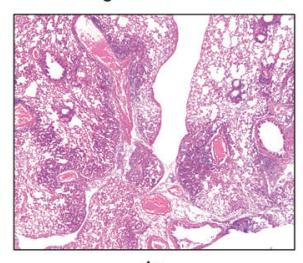

Fig. 3 Knockdown of DSTYK promoted the growth of A549 and SPC-A-1 cells. A Western blot was performed to examine the knockdown efficiency. A549 and SPC-A-1 cells were infected with lentivirus expressing shRNA for DSTYK for 24 hours and selected with puromycin. Then, western blotting was performed. B The MTT assay was performed to examine the effects of DSTYK knockdown on the growth of A549 and SPC-A-1 cells. C, D A colony formation assay was performed, and the results were quantified using control cells and DSTYK-knockdown cells. E-H A tumorigenesis assay was performed to determine the functions of DSTYK in lung cancer. To induce lung cancer, the 8-week-old male KP mice were randomly divided into two groups ( 5 mice in each group) and anesthetized with $2.5 \%$ avertin; full anesthetization and absence of reaction to pain were ensured. A total of $1 \times 10^{9}$ viruses (pSECC/Sg con or pSECC/Sg DSTYK) (OBIO, Shanghai) were administered per mouse using an intranasal/orthotropic infection protocol. The administration of pSECC/Sg con viruses led to the deletion of P53 and activation of $\mathrm{Kras}^{\mathrm{G}}{ }^{2 \mathrm{D}}$. The administration of pSECC/Sg DSTYK viruses led to the deletion of P53 and DSTYK and activation of Kras ${ }^{\mathrm{G}}{ }^{2 \mathrm{D}}$. Twelve weeks later, the mice were killed, lung tissues were collected and photographed, and the tumors were indicated with "*" (E), the tumors were examined with HE staining and analyzed $(\mathbf{F}, \mathbf{G})$, and the expression of DSTYK in the tumors was examined using western blot. The scale bar was indicated. ${ }^{*} P<0.05 ;{ }^{* *} P<0.01$.

TOPFlash reporter (Fig. 5A), the mRNA levels of Axin2 and c-Myc (two targets of $\beta$-catenin) (Fig. 5B) and the accumulation of $\beta$-catenin (Fig. 5C). Similarly, DSTYK overexpression inhibited the expression of target genes downstream of $\beta$-catenin (Fig. 5D). Moreover, DSTYK overexpression elevated the phosphorylation level of $\beta$-catenin (Fig. 5E). Because DSTYK has serine/threonine kinase activity and interacts with $\beta$-catenin, we conducted an in vitro kinase reaction to detect the phosphorylation effect of DSTYK on $\beta$-catenin. The results indicate that DSTYK phosphorylated $\beta$-catenin in vitro (Fig. $5 F$ ).

LDHA is a target gene of the Wnt/ $\boldsymbol{\beta}$-catenin signaling pathway Next, the RNA-seq was performed using A549 control cells and A549 cells with DSTYK knockdown, which suggested that 
A
SPC-A-1

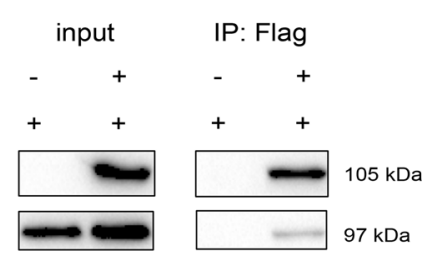

B

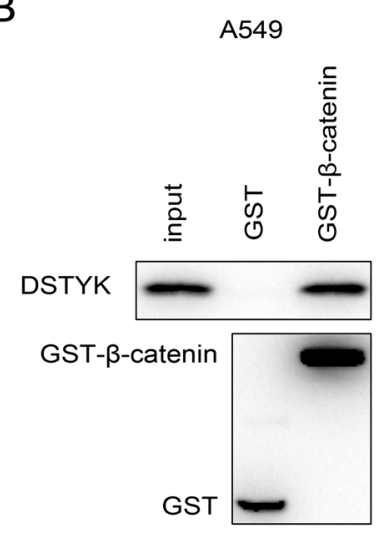

SPC-A-1

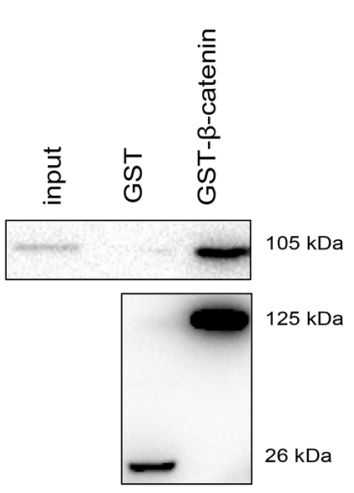

C

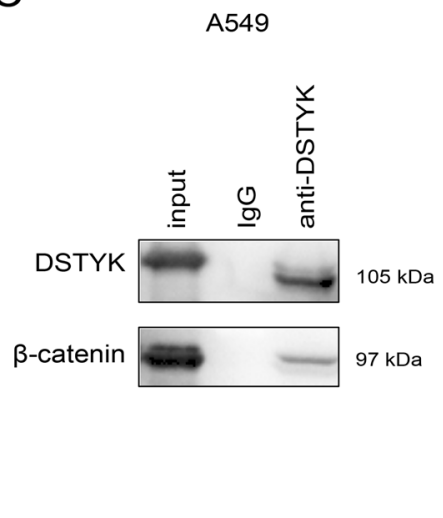

$\mathrm{D}$

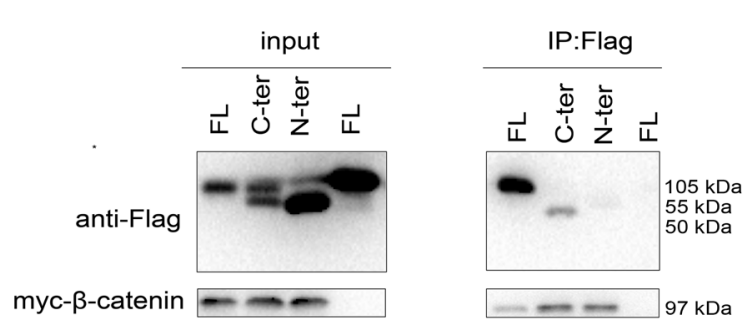

Fig. 4 DSTYK interacts with $\beta$-catenin. A The interaction between exogenously expressed Flag-DSTYK and myc- $\beta$-catenin was examined using immunoprecipitation. Cells were harvested $48 \mathrm{~h}$ after transfection. B A GST pull-down assay was performed to confirm the interaction between endogenous $\beta$-catenin and the fusion protein GST-DSTYK. C The interaction between exogenously expressed Flag-DSTYK and myc$\beta$-catenin was examined using immunoprecipitation. Cells were harvested and lysed with RIPA buffer, and anti- $\beta$-catenin antibody was added for immunoprecipitation. D An immunoprecipitation assay was performed to determine the domain of DSTYK that binds myc- $\beta$-catenin. N-ter the N-terminus of DSTYK (1-500 aa), C-ter the C-terminus of DSTYK (501-929 aa), FL the full length of DSTYK.

knockdown of DSTYK upregulated the mRNA levels of LDHA (Fig. $6 \mathrm{~A}$, The GEO number is GSE178487). Therefore, we next verified the results of RNA-seq. It was found that the LDHA protein level was inhibited by DSTYK overexpression and upregulated by knockdown of DSTYK expression (Fig. 6B). Moreover, we found a binding site for $\beta$-catenin/TCF (TBE, TCF-binding element) in the LDHA promoter region and analyzed the LDHA promoter (Fig. 6C). After TBE was removed or mutated, $\beta$-catenin had no significant activation effect on the LDHA promoter (Fig. 6D). In addition, chromatin immunoprecipitation showed that $\beta$-catenin formed a complex with the LDHA promoter (Fig. $6 \mathrm{E}$ ). These results suggest that LDHA is a target gene of the $W n t / \beta$-catenin signaling pathway.

\section{DSTYK exerts biological functions through Wnt/ $\beta$-Catenin/ LDHA signaling}

We analyzed whether the biological function of DSTYK depends on the Wnt/ $\beta$-catenin/LDHA signaling pathway. In clinical lung cancer specimens, DSTYK protein level was inversely correlated with the expression of $\beta$-catenin and LDHA (Fig. 7A). Moreover, the lactate content was upregulated in lung cancer cells in which
DSTYK expression was disturbed; this upregulation could be restored by knockdown of $\beta$-catenin or LDHA expression (Fig. 7B). In terms of biological function, disturbing DSTYK expression promoted sphere formation, which could be restored in the manner stated above (Fig. 7C). These results indicate that the biological effects of DSTYK occur via Wnt/ $\beta$-catenin/LDHA signaling.

\section{DISCUSSION}

In this study, we found that DSTYK expression was downregulated in lung adenocarcinoma. Upregulation of DSTYK expression in lung adenocarcinoma inhibited the growth of cancer cells, colony formation, and sphere formation; downregulation of DSTYK expression accelerated lung cancer cell growth and colony formation. Additionally, knockout of DSTYK expression in the KP mouse model of lung adenocarcinoma promoted the development of lung cancer. An experiment on the molecular mechanism showed that DSTYK upregulated the level of $\beta$-catenin phosphorylation and inhibited the $\mathrm{Wnt} / \beta$-catenin signaling pathway. Lactate dehydrogenase (LDHA) was found to be a downstream 
A
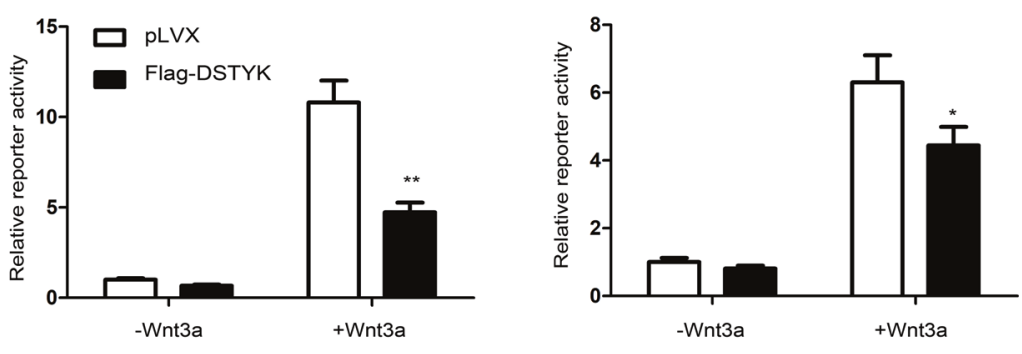

Axin 2

c-Myc
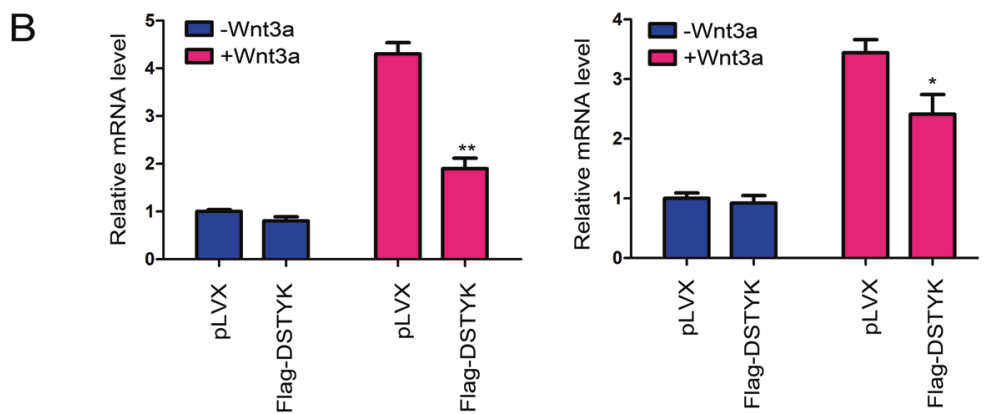

C
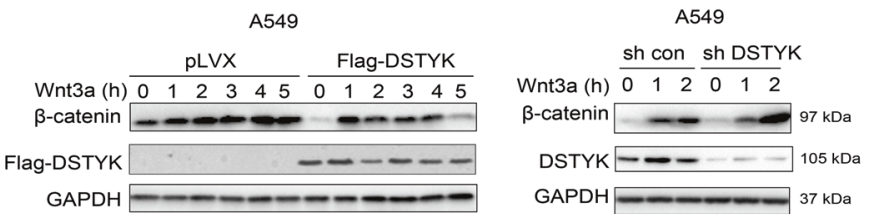

$\mathrm{D}$

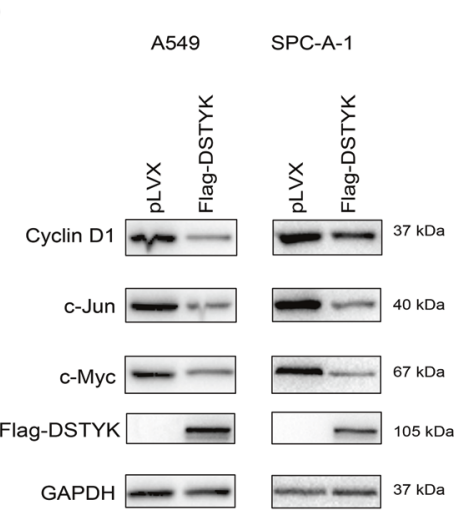

$\mathrm{E}$

$\mathrm{F}$

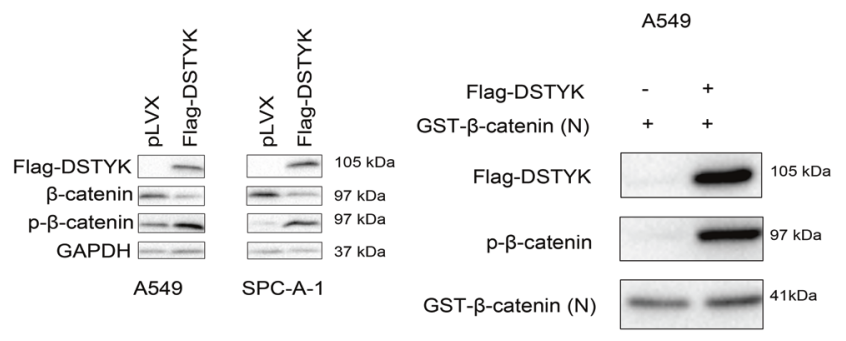

Fig. 5 DSTYK inhibited Wnt/ $\boldsymbol{\beta}$-catenin signaling and promoted the phosphorylation of $\boldsymbol{\beta}$-catenin. A The TOPFlash reporter assay was performed to examine the effects of DSTYK on the transcriptional activity of $\beta$-catenin. B qPCR was performed to examine the effects of DSTYK on the expression of Axin2 and c-Myc. C Western blot was performed to examine the effects of DSTYK on the accumulation of $\beta$-catenin. Control cells and DSTYK-overexpressing cells were treated with Wnt3a for the indicated duration, and the protein levels of $\beta$-catenin were examined. D Western blot was performed to examine the effects of DSTYK on the expression of cyclin D1 and c-Myc. E The phosphorylation of $\beta$-catenin was examined after overexpression of DSTYK. F. In vitro kinase assay. Flag-DSTYK was immunoprecipitated and incubated with GST-N- $\beta$-catenin at $32^{\circ} \mathrm{C}$ for $30 \mathrm{~min}$, and the phosphorylation of $\beta$-catenin was examined. ${ }^{*} P<0.05 ;{ }^{* *} P<0.01$.

target gene of the DSTYK/Wnt/ $\beta$-catenin signaling pathway; thus, the biological function of DSTYK was determined to be exerted through the activation of Wnt/ $\beta$-catenin/LDHA signaling. This study reveals the function and mechanism of DSTYK in lung cancer and suggests the importance of DSTYK agonists in the treatment of lung cancer.
The phosphorylation of $\beta$-catenin by DSTYK is one of the most novel findings in this study. Kinases are important targets for tumor therapy. Previous studies have shown that GSK3 $\beta$ and CK1a are the most important kinases in the Wnt/ $\beta$-catenin signaling pathway during embryonic development [13]. The biological context of tumorigenesis and progression completely 


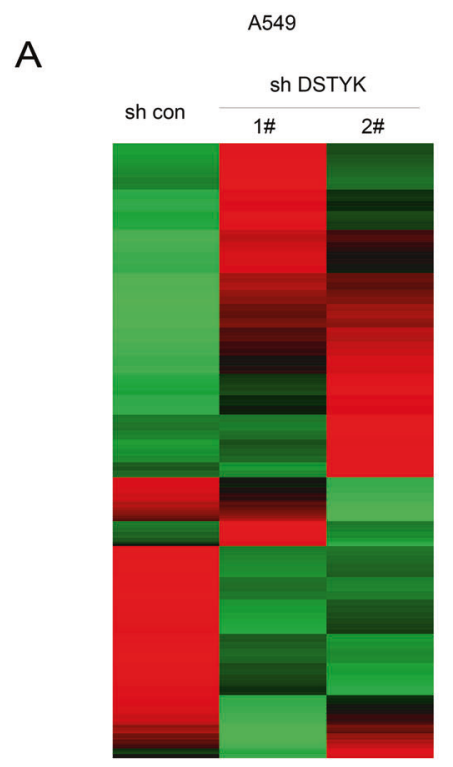

D
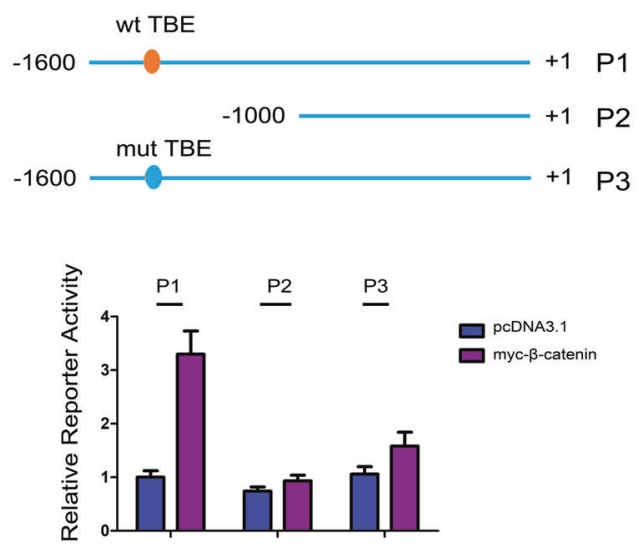

B

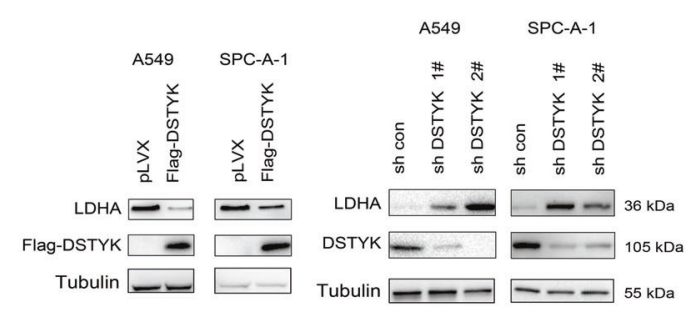

C

GGATCTATCTCAAAGCTGCACT

$-1533 \quad-1528$
$\mathrm{E}$

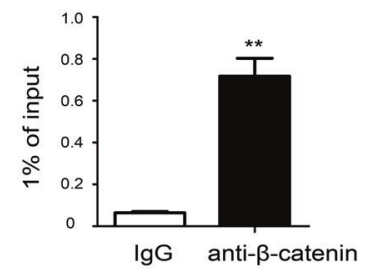

Fig. $6 \boldsymbol{\beta}$-Catenin regulated the expression of LDHA. A The heatmap of the RNA-seq was shown. B The protein levels of LDHA in cells with DSTYK overexpression or knockdown. C The sequence of the human LDHA promoter. D The TBE sequence at -1533 to -1528 in the LDHA promoter was indicated and mutated for the luciferase assay. E ChIP was performed to examine the binding of $\beta$-catenin to the LDHA promoter. ${ }^{* *} P<0.01$.

differs from that of embryonic development. Therefore, it is likely that novel kinases regulate the $\mathrm{Wnt} / \beta$-catenin signaling pathway in tumorigenesis. This study also reveals phosphorylation of amino acid residues $33,37,41$, and 45 of $\beta$-catenin, which contributes to further understanding of the $\mathrm{Wnt} / \beta$-catenin signaling pathway.

The regulation of LDHA expression through the Wnt/ $\beta$-catenin signaling pathway was also found in this study. LDHA catalyzes lactate production, and glycolysis is one of the basic characteristics of tumor cell metabolism [23, 24]. Upon producing lactate, tumor cells quickly obtain intermediate materials for ATP and biomacromolecule synthesis, reshaping the tumor microenvironment [23].

Multiple studies have demonstrated the roles of LDHA in the progression of lung cancer. The expression level of LDHA has been negatively correlated with the survival of lung cancer patients [25]. LDHA has also been shown to mediate the functions of many oncogenes in lung cancer [26]. Treatment of lung cancer cells with lactic acid promotes the migration and infiltration of lung cancer cells by upregulating the expression of snail and inhibits the senescence of lung cancer cells [27]. Moreover, lactic acid upregulates the expression of PD-L1 in lung cancer cells and remodels the immune microenvironment of tumor cells [28]. Consistently, LDHA inhibitor inhibits the tumorigenicity of A549 cells in nude mice [29]. In fact, several studies have revealed that tumor metabolism is regulated by the $W n t / \beta$-catenin signaling pathway. Deng et al. found that the Wnt/ $\beta$-catenin signaling pathway accelerated the progression of pancreatic cancer by directly activating the expression of enzymes (e.g., HMGCR and HMGCS) related to mevalonate metabolism [30]. Furthermore, some reports have shown that PDK1, a key enzyme for glucose metabolism, is a target gene of the $W n t / \beta$-catenin signaling pathway in liver cancer [31]. These studies have demonstrated that the $W n t / \beta$-catenin signaling pathway plays a significant role in the regulation of tumor metabolism.

In summary, this study revealed the expression pattern of DSTYK in lung cancer, clarified its function and mechanism in lung cancer, and provided a new target for the treatment of lung cancer. 

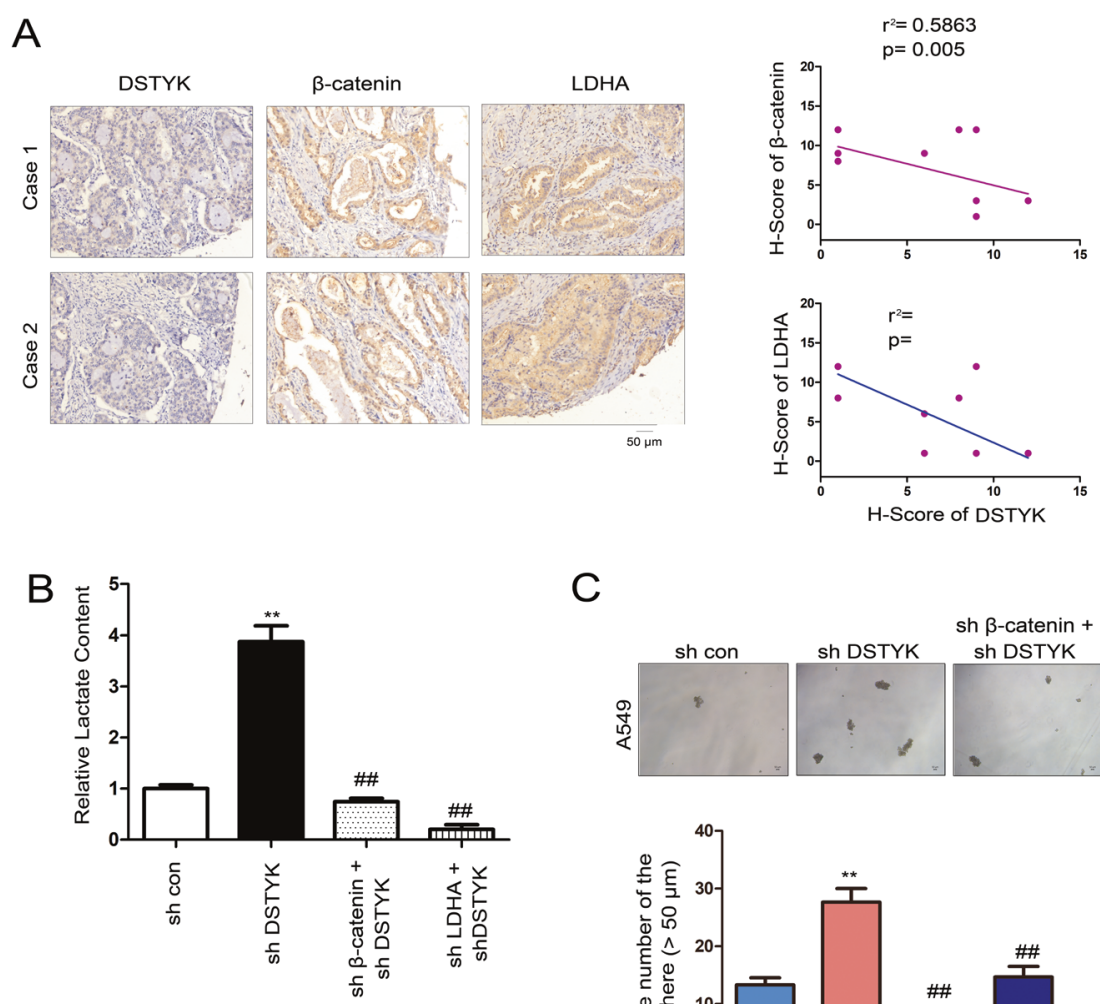

C
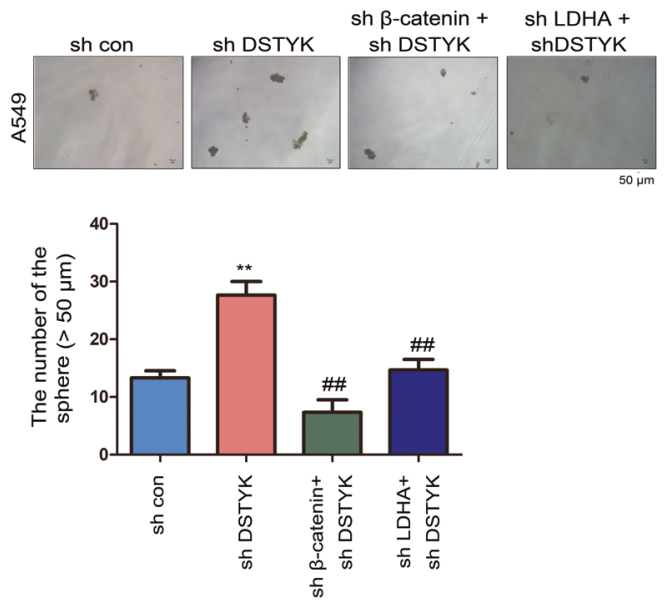

Fig. 7 DSTYK/Wnt/ $\beta$-catenin signaling regulated LDHA expression. A The correlation between DSTYK, $\beta$-catenin and LDHA in clinical lung tissues. The protein levels of DSTYK, $\beta$-catenin and LDHA in 10 lung cancer tissues were examined. The immunostaining was scored. Representative images of two cases are shown, and the correlations between the expression of DSTYK, $\beta$-catenin, and LDHA were analyzed.

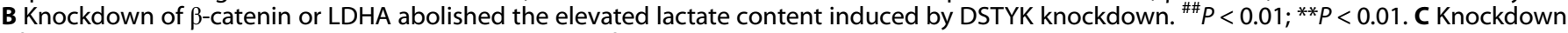
of $\beta$-catenin or LDHA abolished the enhanced sphere formation induced by DSTYK knockdown. The sphere was counted and the statistical analysis was performed. ${ }^{* *} P<0.01 ;{ }^{\# \#} P<0.01$. The scale bar was indicated.

\section{DATA AVAILABILITY}

The datasets used and analyzed during the current study are available from the corresponding author on reasonable request.

\section{REFERENCES}

1. Miller KD, Fidler-Benaoudia M, Keegan TH, Hipp HS, Jemal A, Siegel RL. Cancer statistics for adolescents and young adults, 2020. CA Cancer J Clin. 2020;70:443-59.

2. Siegel RL, Miller KD, Jemal A. Cancer statistics, 2020. CA Cancer J Clin. 2020;70:7-30

3. Romero D. Lung cancer at ASCO20 Virtual. Nat Rev Clin Oncol. 2020;17:450.

4. Riffell JL, Lord CJ, Ashworth A. Tankyrase-targeted therapeutics: expanding opportunities in the PARP family. Nat Rev Drug Discov. 2012;11:923-36.

5. Li F, Yang $H$, Kong $T$, Chen $S$, Li $P$, Chen $L$, et al. PGAM1, regulated by miR-3614$5 p$, functions as an oncogene by activating transforming growth factor-beta (TGF-beta) signaling in the progression of non-small cell lung carcinoma. Cell Death Dis. 2020;11:710.

6. Villanueva MT. Lung cancer: whack a GLI Hedgehog. Nat Rev Clin Oncol. 2014;11:121.

7. Luengo A, Abbott KL, Davidson SM, Hosios AM, Faubert B, Chan SH, et al. Reactive metabolite production is a targetable liability of glycolytic metabolism in lung cancer. Nat Commun. 2019;10:5604.

8. Sarode P, Zheng X, Giotopoulou GA, Weigert A, Kuenne C, Gunther S, et al. Reprogramming of tumor-associated macrophages by targeting beta-catenin/ FOSL2/ARID5A signaling: a potential treatment of lung cancer. Sci Adv. 2020;6: eaaz6105.
9. Kolegova ES, Shashova EE, Kostromitskii DN, Dobrodeev AY, Kondakova IV. Betacatenin in non-small cells lung cancer and its association with proteasomes. Bull Exp Biol Med. 2020;168:677-80.

10. $\mathrm{Xu} \mathrm{H}$, Lin D, Wang L, Liu N, Wang E. Expression and mutation of beta-catenin in non-small cell lung cancer. Zhongguo fei ai za zhi. 2004;7:409-13.

11. Perugorria MJ, Olaizola $P$, Labiano I, Esparza-Baquer A, Marzioni M, Marin JJG, et al. Wnt-beta-catenin signalling in liver development, health and disease. Nat Rev Gastroenterol Hepatol. 2019;16:121-36.

12. Kypta RM, Waxman J. Wnt/beta-catenin signalling in prostate cancer. Nat Rev Urol. 2012;9:418-28.

13. Liu C, Li Y, Semenov M, Han C, Baeg GH, Tan Y, et al. Control of beta-catenin phosphorylation/degradation by a dual-kinase mechanism. Cell. 2002;108: 837-47.

14. Peng J, Dong W, Chen Y, Mo R, Cheng JF, Hui CC, et al. Dusty protein kinases: primary structure, gene evolution, tissue specific expression and unique features of the catalytic domain. Biochim Biophys Acta. 2006;1759:562-72.

15. Bagwell J, Norman J, Ellis K, Peskin B, Hwang J, Ge X, et al. Notochord vacuoles absorb compressive bone growth during zebrafish spine formation. eLife. 2020;9:e51221.

16. Sun X, Zhou Y, Zhang R, Wang Z, Xu M, Zhang D, et al. Dstyk mutation leads to congenital scoliosis-like vertebral malformations in zebrafish via dysregulated mTORC1/TFEB pathway. Nat Commun. 2020;11:479.

17. Boissel S, Fallet-Bianco C, Chitayat D, Kremer V, Nassif C, Rypens F, et al. Genomic study of severe fetal anomalies and discovery of GREB1L mutations in renal agenesis. Genet Med. 2018;20:745-53.

18. Payton S. Genetics: DSTYK gene linked to urinary tract defects. Nat Rev Urol. 2013;10:492. 
19. Sanna-Cherchi S, Sampogna RV, Papeta N, Burgess KE, Nees SN, Perry BJ, et al. Mutations in DSTYK and dominant urinary tract malformations. N Engl J Med. 2013;369:621-9.

20. Li K, Liu JW, Zhu ZC, Wang HT, Zu Y, Liu YJ, et al. DSTYK kinase domain ablation impaired the mice capabilities of learning and memory in water maze test. Int J Clin Exp Pathol. 2014;7:6486-92.

21. Tang G, Yang Y, Shang L, Jun F, Liu Q. A DSTYK mutation activates ERK1/2 signaling to promote intraspinal dissemination in a case of solitary fibrous tumor/ hemangiopericytoma. Lab Invest. 2019;99:1501-14.

22. Wu Q, Tian $\mathrm{Y}$, Zhang J, Tong $\mathrm{X}$, Huang $\mathrm{H}$, Li S, et al. In vivo CRISPR screening unveils histone demethylase UTX as an important epigenetic regulator in lung tumorigenesis. Proc Natl Acad Sci USA. 2018;115:E3978-E86.

23. Yu H, Yin Y, Yi Y, Cheng Z, Kuang W, Li R, et al. Targeting lactate dehydrogenase $A$ (LDHA) exerts antileukemic effects on T-cell acute lymphoblastic leukemia. Cancer Commun. 2020;40:501-17.

24. Liang T, Ye X, Yan D, Deng C, Li Z, Tian B. FAM46B promotes apoptosis and inhibits glycolysis of prostate cancer through inhibition of the MYC-LDHA axis. OncoTargets Ther. 2020;13:8771-82.

25. Yu C, Hou L, Cui H, Zhang L, Tan X, Leng X, et al. LDHA upregulation independently predicts poor survival in lung adenocarcinoma, but not in lung squamous cell carcinoma. Future Oncol. 2018;14:2483-92.

26. Yin D, Hua L, Wang J, Liu Y, Li X. Long non-coding RNA DUXAP8 facilitates cell viability, migration, and glycolysis in non-small-cell lung cancer via regulating HK2 and LDHA by inhibition of miR-409-3p. OncoTargets Ther. 2020;13:7111-23.

27. Li X, Zhang Z, Zhang Y, Cao Y, Wei H, Wu Z. Upregulation of lactate-inducible snail protein suppresses oncogene-mediated senescence through p16(INK4a) inactivation. J Exp Clin Cancer Res. 2018;37:39.

28. Feng J, Yang $\mathrm{H}$, Zhang $\mathrm{Y}$, Wei $\mathrm{H}$, Zhu $\mathrm{Z}$, Zhu B, et al. Tumor cell-derived lactate induces TAZ-dependent upregulation of PD-L1 through GPR81 in human lung cancer cells. Oncogene 2017;36:5829-39.

29. Li XM, Xiao WH, Zhao HX. Discovery of potent human lactate dehydrogenase $A$ (LDHA) inhibitors with antiproliferative activity against lung cancer cells: virtual screening and biological evaluation. MedChemComm 2017;8:599-605.

30. Deng YZ, Cai Z, Shi S, Jiang H, Shang YR, Ma N, et al. Cilia loss sensitizes cells to transformation by activating the mevalonate pathway. J Exp Med. 2018;215:177-95.

31. Zuo Q, He J, Zhang S, Wang H, Jin G, Jin H, et al. PGC1alpha suppresses metastasis of HCC by inhibiting Warburg effect via PPARgamma-dependent WNT/beta-catenin/PDK1 axis. Hepatology. 2021;73:644-60.

\section{AUTHOR CONTRIBUTIONS}

FY and WF performed study concept and design; $C Z, M C$, and $Y C$ performed the experiment, interpreted, and analyzed the data. $C Z$ wrote the paper; $M C$ and $Y C$ prepared the figures; FY provided facilities and material support. WF and FY revised the manuscript; All authors read and approved the final paper.

\section{FUNDING INFORMATION}

The present study was supported by the National Science Foundation of China (grant no. 81401933) and Nurture Projects for Basic Research of Shanghai Chest Hospital (grant no. 2020YNJCM11).

\section{COMPETING INTERESTS}

The authors declare no competing interests.

\section{ETHICS STATEMENT}

All experiments were approved by the Ethics Committee of Shanghai Chest Hospital in accordance with the Declaration of Helsinki. Patients provided written informed consent and the study procedures were fully explained before study inclusion.

\section{ADDITIONAL INFORMATION}

Supplementary information The online version contains supplementary material available at https://doi.org/10.1038/s41419-021-04385-1.

Correspondence and requests for materials should be addressed to Feng Yao or Wentao Fang.

Reprints and permission information is available at http://www.nature.com/ reprints

Publisher's note Springer Nature remains neutral with regard to jurisdictional claims in published maps and institutional affiliations.

(i) Open Access This article is licensed under a Creative Commons Attribution 4.0 International License, which permits use, sharing, adaptation, distribution and reproduction in any medium or format, as long as you give appropriate credit to the original author(s) and the source, provide a link to the Creative Commons license, and indicate if changes were made. The images or other third party material in this article are included in the article's Creative Commons license, unless indicated otherwise in a credit line to the material. If material is not included in the article's Creative Commons license and your intended use is not permitted by statutory regulation or exceeds the permitted use, you will need to obtain permission directly from the copyright holder. To view a copy of this license, visit http://creativecommons. org/licenses/by/4.0/.

(c) The Author(s) 2021 